\title{
Formation of porous membranes for drug delivery systems
}

\author{
P. van de Witte ${ }^{a}$, H. Esselbrugge ${ }^{a}$, A.M.P. Peters ${ }^{a}$, P.J. Dijkstra ${ }^{a}$, J. Feijen ${ }^{a}$, R.J.J. \\ Groenewegen $^{\mathrm{b}}$, J. Smid ${ }^{\mathrm{b}}$, J. Olijslager ${ }^{\mathrm{b}}$, J.M. Schakenraad ${ }^{\mathrm{c}}$, M.J.D. Eenink ${ }^{\mathrm{d}}$ and A.P. Sam ${ }^{\mathrm{d}}$ \\ aniversity of Twente, Department of Chemical Technology, Enschede. The Netherlands \\ ${ }^{-} T N O$ Plastics and Rubber Research Institute, Delf, The Netherlunds \\ 'University of Groningen, Department of Histology and Cell Biolog!, Groningen. The Netherlands

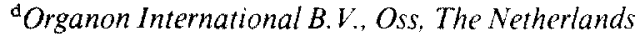

(Received April 1992; accepted in revised form 15 December 1992)

Highly crystalline porous hollow poly (L-lactide) (PLLA) fibres suitable for the delivery of various drugs were obtained using a dry-wet spinning process. The pore structure of the fibres could be regulated by changing the spinning systems and spinning conditions. Using the spinning system PLLAdioxane-water, fibres with a dense toplayer and a spongy sublayer were obtained. The spinning system PLLA-chloroform/toluene-methanol yielded fibres with a very open porous structure. The membrane formation of the former system probably occurs by liquid-liquid demixing followed by crystallization of the polymer rich phase. In the membrane formation process of the spinning system, PLLA-chloroform/toluene-methanol crystallization probably plays a dominant role. The membrane formation process will be related to basic principles of phase separation. The fibres are suitable for the long term zero order delivery of the contraceptive 3-ketodesogestrel and the short term zero order delivery of the cytostatic agent, cisplatin. The drugs are released by dissolution of the drug crystals in the fibre core followed by diffusion through the membrane structure. Short term release of adriamycin could be obtained through an adsorption-desorption mechanism. The pore structures of the fibres have a large influence on the release rates of the drugs investigated. When fibres with dense toplayers were used, low release rates of drugs were observed whereas fibres with well interconnected pore structures over the fibre wall showed very high release rates.

Key words: Poly (L-lactide) fibre; Membrane formation; Dry-wet spinning; Adriamycin; 3-Ketodesogestrel; Cisplatin; Controlled release

\section{Introduction}

In order to improve the effectiveness of drug therapy and to reduce possible systemic side effects, many attempts have been made to deliver

Correspondence to: Professor Dr J. Feijen, Faculty of Chemical Technology, P.O. Box 217, 7500 AE Enschede, The Netherlands. drugs in a controlled way to the human body. Bioresorbable drug delivery devices, especially, have received much attention because there is no need to remove the implant after complete delivery of the drug $[1,2]$. Bioresorbable polymerdrug combinations for drug delivery can be processed either as microparticles or as implants (e.g., films, capsules, needles or pellets). For most systems, release rates can be adjusted by 
using polymers with different chemical composition and physical properties [3].

Recently the development of porous degradable reservoir type release devices has been described. Schindler et al. [4] developed a tubular implant of poly ( $\epsilon$-caprolactone) (PCL) with a porous wall suitable for the release of decapeptides. The tube was prepared by extrusion of the molten PCL to which a certain amount of Pluronic F-68 was added. By treatment of the extruded tube with appropriate solvents, the additive was removed leading to the formation of a reservoir wall with an interconnected pore structure. However this technique does not allow the formation of highly porous walls with interconnected pores of very small sizes $(<1 \mu \mathrm{m})$.

Porous structures can also be obtained using phase separation techniques. Eenink et al. [5] introduced a release system based on the use of poly (L-lactide) (PLLA) fibres produced by a dry-wet spinning process. By applying different spinning conditions, the fibre properties could be influenced to a large extent which gave the possibility to adequately adjust payloads and release rates. The release rate of levonorgestrel appeared to be dependent on the wall membrane structure as was shown in preliminary in vitro release experiments. However for the release of different active compounds at a relatively high ratc, a fibre with a highly porous fibre wall appeared to be necessary.

The development of highly porous fibres with optimal release characteristics requires a thorough knowledge of membrane formation processes. Because of the complexity of the membrane formation process it is difficult to predict the structure that will be obtained using a particular membrane forming combination. Much research has already been dedicated to this subject but a lot of questions remain unanswered. In this study we give an overview of the basic principles of phase separation processes and in particular of the membrane formation process. The concept of membrane formation will be applied to the preparation of poly (L-lactide) hollow fibres. Special attention will be given to the influence of the membrane structure on the release proper- ties of the fibres. The suitability of PLLA fibres as controlled release devices for the delivery of the contraceptive 3-ketodesogestrel and the cytostatic agents cisplatin and adriamycin will be demonstrated.

\section{Theory of membrane formation}

Porous structures are relatively easily obtained using phase separation techniques. Two strategies can be used to demix a polymer solution. The first method makes use of the fact that the thermodynamic quality of a polymer solution usually decreases when the temperature is decreased. This technique is called thermally induced phase separation (TIPS). Thermally induced phase separation is a technique also used frequently in biomedical engineering, for example this principle has been used for the production of wound dressings and artificial arteries $[6,7]$. Another method of obtaining porous structures is phase separation by immersion precipitation. A polymer solution is cast as a thin film on a glass plate or extruded through an annular die and is subsequently immersed in a nonsolvent bath. Demixing can occur because the good solvent in the polymer solution is exchanged for the nonsolvent in the coagulation bath. Both the TIPS process and immersion precipitation make use of the same principles. Because of the simplicity of the former process the principles of structure formation will initially be discussed on the basis of the TIPS process. Later the principles will be extended to the membrane formation process.

\section{Thermally induced phase separation}

A typical phase diagram for a binary polymersolvent system is schematically represented in Fig. 1 [8]. In this figure the temperature is plotted as function of the polymer concentration. At higher temperatures the solution is still homogeneous, at lower temperatures liquid-liquid phase separation in a polymer poor and a polymer rich phase can take place. The miscibility gap can be divided into three areas. The border of 


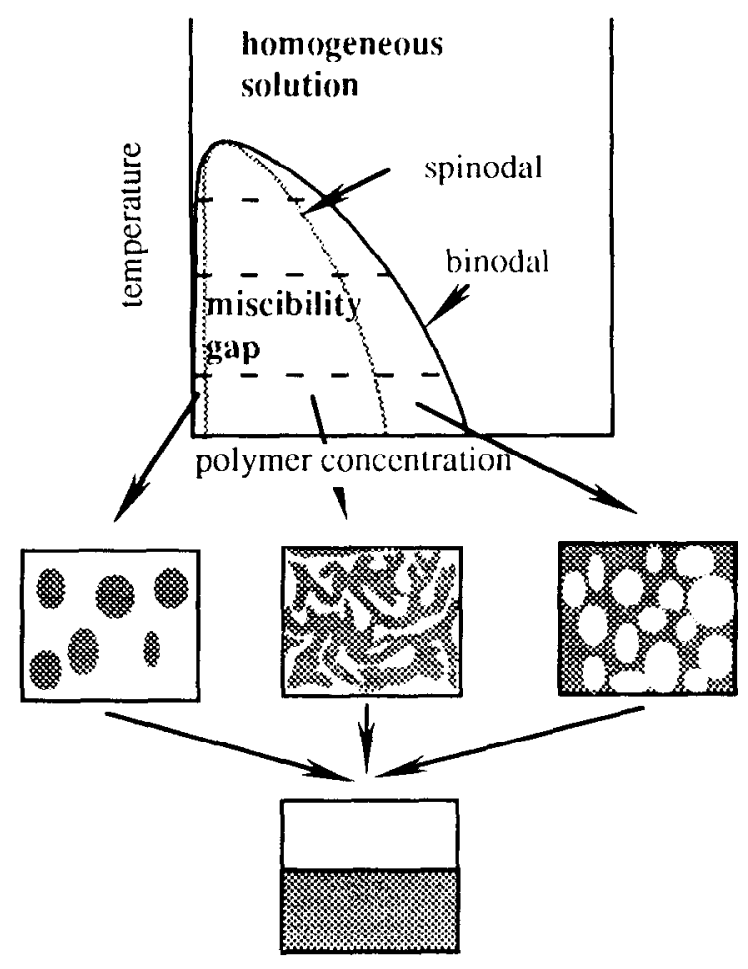

Fig. 1. General phase diagram of a monodisperse polymere solvent combination. A schematical representation of the TIPS process is given showing the structures that are usually found when a homogeneous solution is quenched into the miscibility gap. At low polymer concentrations the demixing process can given latex structures, at high polymer concentrations spongy structures can be obtained and at intermediate concentrations bicontinuous network structures can be formed. When the structure is not stabilized two fully separated layers are obtained.

the miscibility gap is called the binodal, the dashed line is called the spinodal. Examples of compositions that are in equilibrium are given by horizontal lines called tielines. The point where the binodal and spinodal touch is called the critical point. At the critical point the polymer rich phase and the polymer poor phase have the same compositions. Compositions located between the binodal and the spinodal are metastable, this means that solutions are stable with respect to small composition fluctuations. However when the composition fluctuations are large enough phase separation will take place. Phase separation takes place by nucleation and growth of a polymer poor phase in case the original com- position of the solution is located at polymer concentrations higher than the critical point and by nucleation and growth of the polymer rich phase in case the polymer concentration is lower than the polymer concentration of the critical point. Solutions quenched into the area enclosed by the spinodal are unstable [9]. Any composition fluctuation can trigger a wave of composition fluctuations through the solution. The amplitude of the fluctuations will increase because the molecules of both components will move from low concentration regions to high concentration regions till the compositions of the polymer rich and the polymer poor phase approach the binodal compositions.

Due to the tendency to minimize the interfacial energy the structures will coarsen in time and eventually two fully separated layers may be obtained. Several ways are possible to stabilize the structure formed during the demixing process. The main principle of these techniques is to continue cooling the solution till another stabilizing transition is passed. The phase transitions encountered most often are vitrification, crystallization of the polymer or freezing of the solvent [10-13]. Afterwards the solvent can be removed by evaporation, freeze drying, extraction with non-solvents or critical point drying. The theoretical phase diagrams combining liquidliquid demixing and the fixation possibilities are given in Fig. 2.

The most elegant technique is vitrification of the polymer solution (Fig. 2a) $[10,11]$. The structure formed during the demixing process is 'frozen in' without the interference of an other structure inducing transition. During the demixing process the polymer rich phase is vitrified. At low polymer concentrations a latex is obtained (nucleation and growth of polymer rich phase, see Fig. 1). At high polymer concentrations a spongy structure is obtained (nucleation and growth of polymer poor phase). When the solution is quenched rapidly through the metastable area the unstable area can be reached. Spinodal demixing usually yields network structures, both the polymer poor phase and the polymer rich phase form continuous structures. The structure 


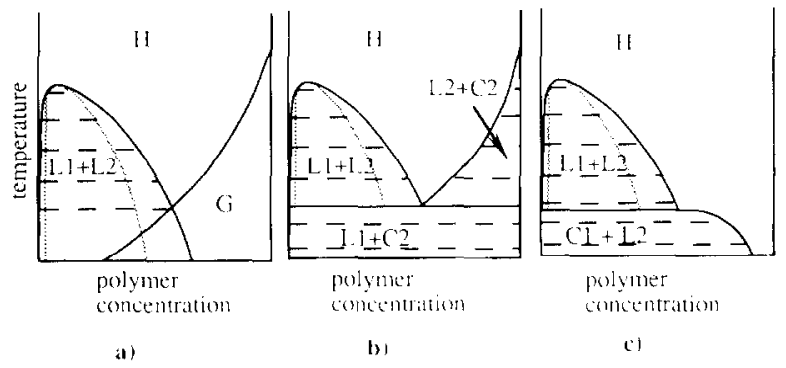

Fig. 2. Equilibrium phase diagrams for monodisperse polymer-solvent systems. (a) Combination of liquid-liquid demixing and a glass transition; (b) combination of liquid-liquid demixing and crystallization of the polymer; (c) combination of liquid-liquid demixing and crystallization of the solvent. Symbols: L1, liquid phase with a relatively low polymer concentration; $\mathrm{L} 2$. liquid phase with a relatively high polymer concentration; $\mathrm{C} 1$, crystalline phase with a low polymer concentration; $\mathrm{C} 2$, crystalline phase with a high polymer concentration; $G$ indicates the glassy state.

can also be stabilized by polymer crystallization (Fig. 2b). At higher polymer concentrations porous structures can be obtained by nucleation and growth of crystallites [12]. At lower concentrations the polymer rich phase formed after liquidliquid demixing can crystallize. The kinetics of demixing can play an important role in these processes. Solvent crystallization also is an effective means of stabilizing the demixed solution (Fig. 2c). Contrary to vitrification, crystallization processes can strongly influence the structure. Important variables in the TIPS process are polymer concentration, polymer-solvent interaction, molecular weight and molecular weight distribution of the polymer, viscosity of the solvent and the cooling program.

\section{Membrane formation by immersion precipitation}

Immersion precipitation is more difficult to understand because at least three components are involved and complex diffusion processes play an important role. Immersion precipitation can be regarded as an isothermal process, so the compositions can be plotted in a triangle. The corners represent the pure components, the axes the three binary combinations and a point within the triangle a ternary composition (Fig. 3). In principle, the same three parts of the miscibility

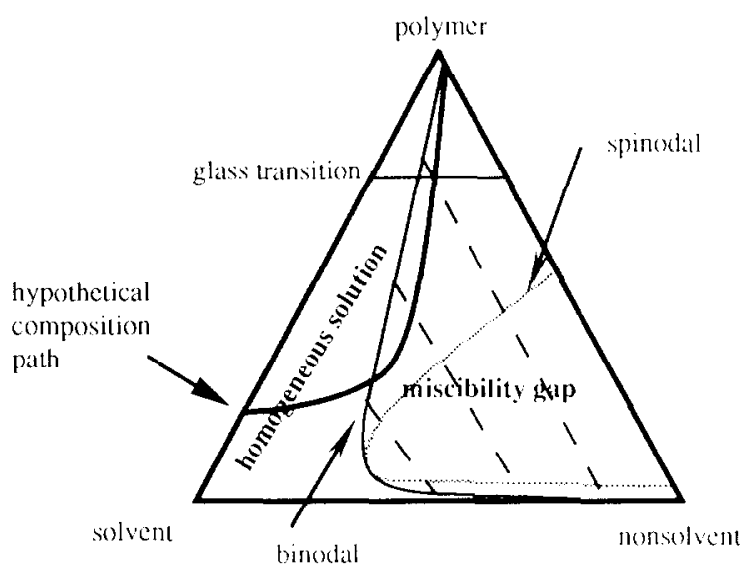

Fig. 3. Isothermal ternary phase diagram of a ternary solution containing a polymer, a solvent and a non-solvent. A schematic representation of a hypothetical composition path that is followed by an element in the polymer solution during the membrane formation process is given. After the composition has entered the miscibility gap phase separation in a polymer rich and a polymer poor phase takes place. In this figure only the composition path of the polymer rich phase is given. After the composition has reached the glassy state the membrane structure is fixed.

gap are present as in the binary diagram. However the critical point is usually located at low polymer concentrations and normally is not reached [14]. It has also been shown that spinodal demixing by diffusional processes is not very likely [15]. Phase separation usually takes place by nucleation and growth of a polymer poor phase. Several models have been developed to describe the diffusion process between immersion and phase separation [14-16]. Basically two different types of exchange processes are encountered. Solutions that demix instantaneously after immersion and solutions that show a delay time before demixing [14]. It has been shown that the solvent-nonsolvent interaction and the mutual diffusion coefficients are the determining parameters. When the solvent-nonsolvent exchange is very rapid the composition path enters the miscibility gap almost immediately after immersion. When the exchange rate of the solvent and the non-solvent is moderate delayed demixing takes place, the composition of the solution remains in the homogeneous part of the phase diagram for a certain time. During this de- 
lay time the compositions in the polymer solution gradually shift to higher non-solvent concentrations until finally the miscibility gap is entered. Also during the demixing process the exchange of solvent for nonsolvent continues. Both the compositions of the polymer rich phase and the polymer poor phase will move towards the polymer-nonsolvent axis (Fig. 3 ). For amorphous polymers the membrane structures can be stabilized by very high viscosities in the polymer rich phase or by reaching the glassy state [17]. The two different exchange regimes also result into two different types of membrane structures. Rapidly demixing solutions usually have a thin, rather dense top layer and a very open cell structure in the sublayer. Solutions that show delayed demixing usually have a thick dense top layer and a sublayer with cells which are less interconnected [14]. The low porosity of the top layer can probably be related to the high polymer concentration in the interfacial layer between the polymer solution and the coagulation bath at the moment demixing takes place.

When crystallization of the polymer can also take place the phase diagram becomes very complex [18]. Often crystallization processes are too slow to play a role in the rapid membrane formation process and membranes are obtained that are not crystalline at all. However systems are also known where crystallization is more rapid than liquid-liquid demixing or where crystallization takes place after the liquid-liquid demixing process has generated a high polymer concentration; examples of these systems will be given in this article. Parameters that strongly influence the structure of the membrane are the composition of the polymer solution, the composition of the coagulation bath and the choice of the solvent and the nonsolvent. For example addition of nonsolvent to the polymer solution will speed up the demixing process, the thickness of the toplayer will decrease and the porosity of the sublayer will usually increase. When solvent is added to the coagulation bath it is possible to obtain membranes without a toplayer [26].

Often additives are used to obtain the desired structurc. Sometimes morc solvents or nonsol- vents are introduced to the polymer solution. Also so called porosifiers like poly ( $N$-vinylpyrrolidone ) and polyethyleneglycol are often used $[5,19]$. In fact the ternary systems are then abandoned for a quaternary system and predictions and explanations of structures are becoming tedious.

\section{Materials and Methods}

\section{Materials}

Poly (L-lactide) (PLLA) was purchased from Purac Biochem BV, Gorinchem, The Netherlands. Viscosity measurements at $25^{\circ} \mathrm{C}$ of the PLLA, dissolved in chloroform, gave an intrinsic viscosity of $4.7 \mathrm{gl} / \mathrm{g}$. Because of the high viscosity of the solutions with high polymer concentrations the intrinsic viscosity of the PLLA used for the phase diagrams was $2 \mathrm{dl} / \mathrm{g}$. The chloroform used for the phase diagrams was of chromatographic grade (Lichrosolv, Merck-Schuchard, Darmstadt, Germany). The chloroform used for the other experiments was of analytical grade (Merck-Schuchard). Solvents and nonsolvents (toluene, $n$-hexane, 1,4-dioxane, methanol, ethanol) used for the preparation of solutions and for the production of hollow fibres, were of analytical grade (Merck-Schuchardt). Methanol used as an external coagulant in the dry-wet spinning process was of technical quality (UCB). Water was demineralized and ultrafiltrated. Poly (vinyl alcohol) (PVA, 88\% hydrolysed, molecular weight $15000 \mathrm{~g} / \mathrm{mol}$ (as given by the supplier)) was purchased from Janssen Pharmaceutica, Beerse, Belgium. 3-Ketodesogestrel (3-KD) was supplied by Organon International BV, Oss, The Netherlands. Cisplatin was supplied by Johnson Matthey GmbH Alfa Products (Karlsruhe, Germany). Adriamycin (Adx) was obtained from Farmitalia, Milan, Italy. All other chemicals were obtained from Merck-Schuchardt, Darmstadt, Germany.

\section{Dry-wet spinning procedure}

The hollow fibre dry-wet spinning process is schematically shown in Fig. 4. The spinning so- 


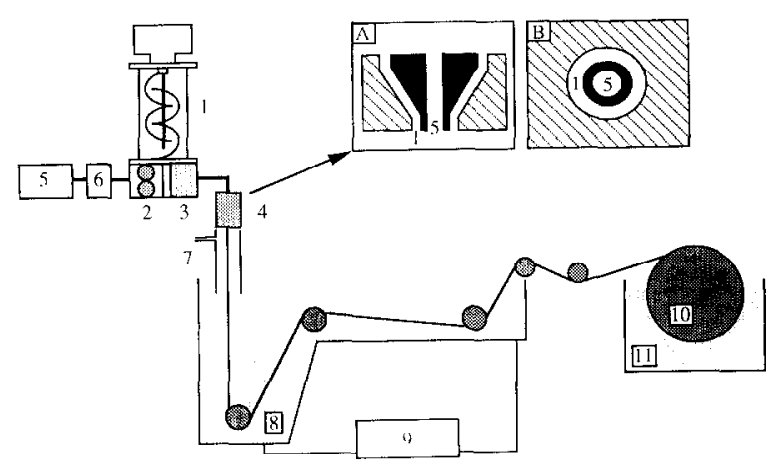

Fig. 4. Experimental set up for the dry-wet spinning process. 1. Polymer solution. 2. Gear pump. 3. Metal filter. 4. Spinneret. 5. Bore liquid. 6. HPLC-pump. 7. Air gap. 8. External coagulation bath. 9 . Temperature control. 10. Collecting devicc. 11. Flushing bath.

lution was prepared in the solution reservoir (1). The temperature of the solution usually was between 50 and $65^{\circ} \mathrm{C}$. The spinning solution was degassed and was pumped (2) from the reser- voir through a metal filter ( 3 ; mesh size $40 \mu \mathrm{m}$ ) into the spinneret ( 4 ; tube-in-orifice device: orifice diameter of $0.8 \mathrm{~mm}$; injection tube outside and inside diameter of 0.6 and $0.4 \mathrm{~mm}$, respectively). The temperature of the spinneret could be adjusted between 20 and $50^{\circ} \mathrm{C}$. The internal coagulant (5) was delivered pulse-free with a HPLC pump (6) to the inner tube of the spinneret. The spinneret was positioned at a distance $h$ (spinning height) above the external coagulation bath (8). In this air-gap a controlled atmosphere could be maintained ( 7 ). The nonsolvent in the coagulation bath was circulated (9) and maintained at a constant temperature (usually $25^{\circ} \mathrm{C}$ ). After leaving the first bath, the fibre was transferred onto a collecting device (10) placed in a second bath containing the same nonsolvent at room temperature. The speed of the collecting device was adjustable.

PLLA hollow fibres with different properties were prepared from two different spinning sys-

TABLE 1

Spinning systems

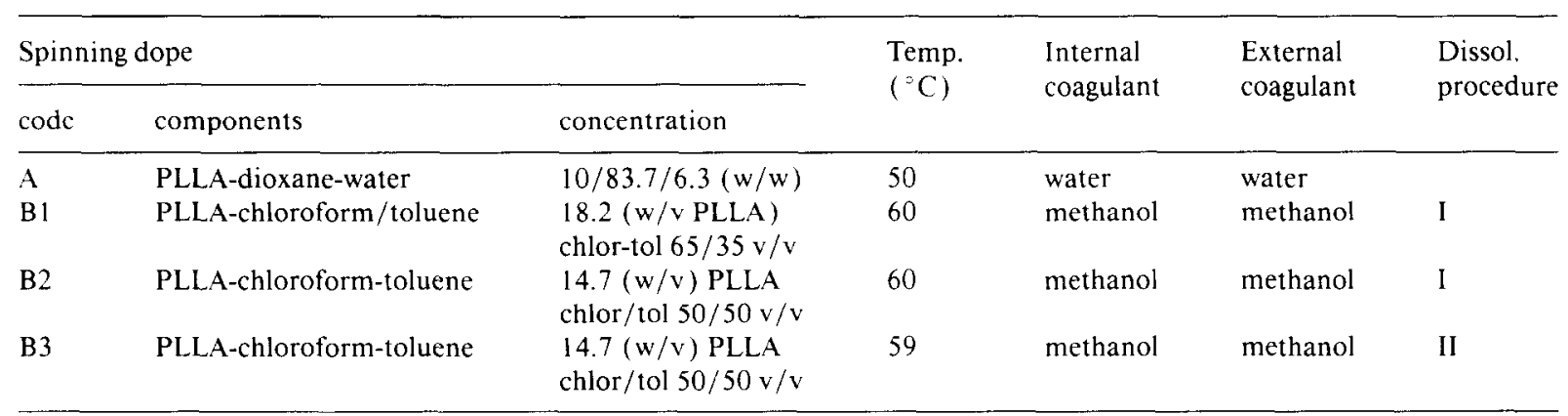

TABLE 2

Spinning conditions applied for the different spinning systems

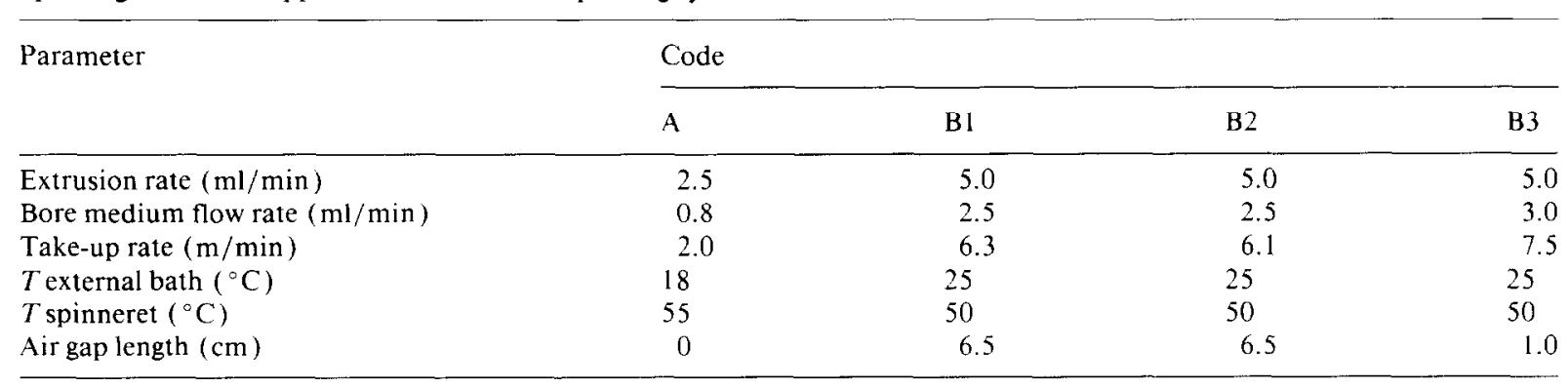




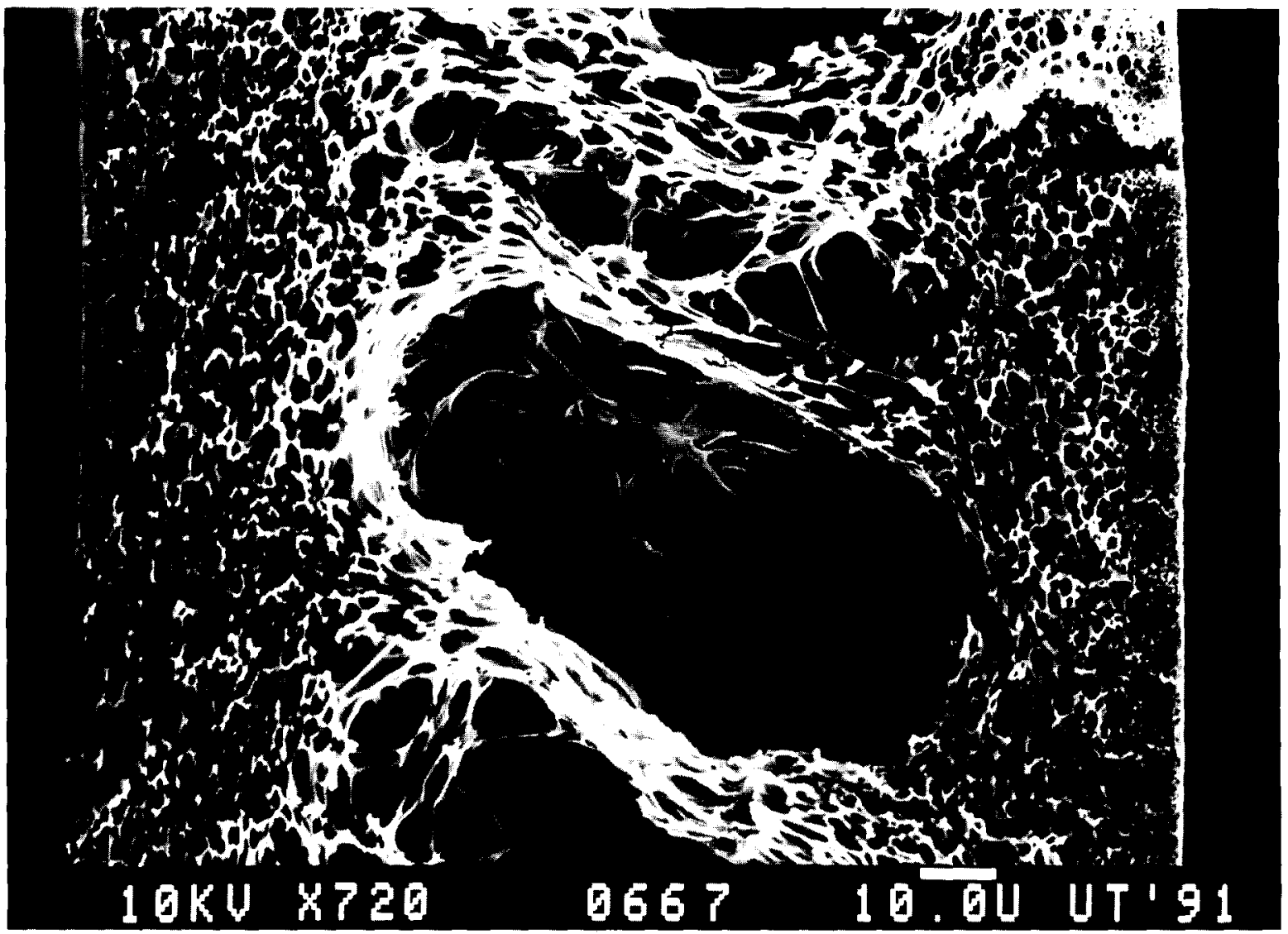

Fig. 5. Scanning electron micrograph of a cross-section of a PI.LA hollow fibre membrane. Outer toplayer at the right hand side Spinning system: PLLA-dioxane-water (system A).

tems. The spinning systems and the relevant spinning conditions are summarized in Tables 1 and 2 , respectively. With respect to fibre preparation from the system PLLA-chloroform/toluene-methanol, it appeared that the applied dissolution procedure strongly influenced the membrane structure of the fibre. Therefore two different procedures were applied. In procedure $I$, the PLLA was first dissolved in a part of the total amount of chloroform needed. After dissolution of the PLLA, a mixture of toluene and the remaining amount of chloroform was added to the highly viscous solution. In procedure II, the PLLA was dissolved directly in a mixture of chloroform and toluene.

Unless mentioned otherwise, after spinning a standard rinsing and drying procedure was ap- plied. The hollow fibre was collected and rinsed for $24 \mathrm{~h}$ in a bath containing a large amount of fresh nonsolvent. If the external coagulation bath contained methanol, the rinsed fibres were transferred to a bath containing $n$-hexane to replace methanol during another period of $24 \mathrm{~h}$. If the external coagulant was water, the water was first replaced by ethanol ( 24 hours) followed by immersion in $n$-hexane for another $24 \mathrm{~h}$. After the rinsing procedure, the fibres were air-dried and dried overnight in vacuo at ambient temperature. After spinning, the fibres were characterized with respect to microstructure and dimensions using scanning electron microscopy. Samples of the fibres were wetted in ethanol and fractured in liquid nitrogen. The ethanol was subsequently exchanged for $n$-hexane. The fibres 


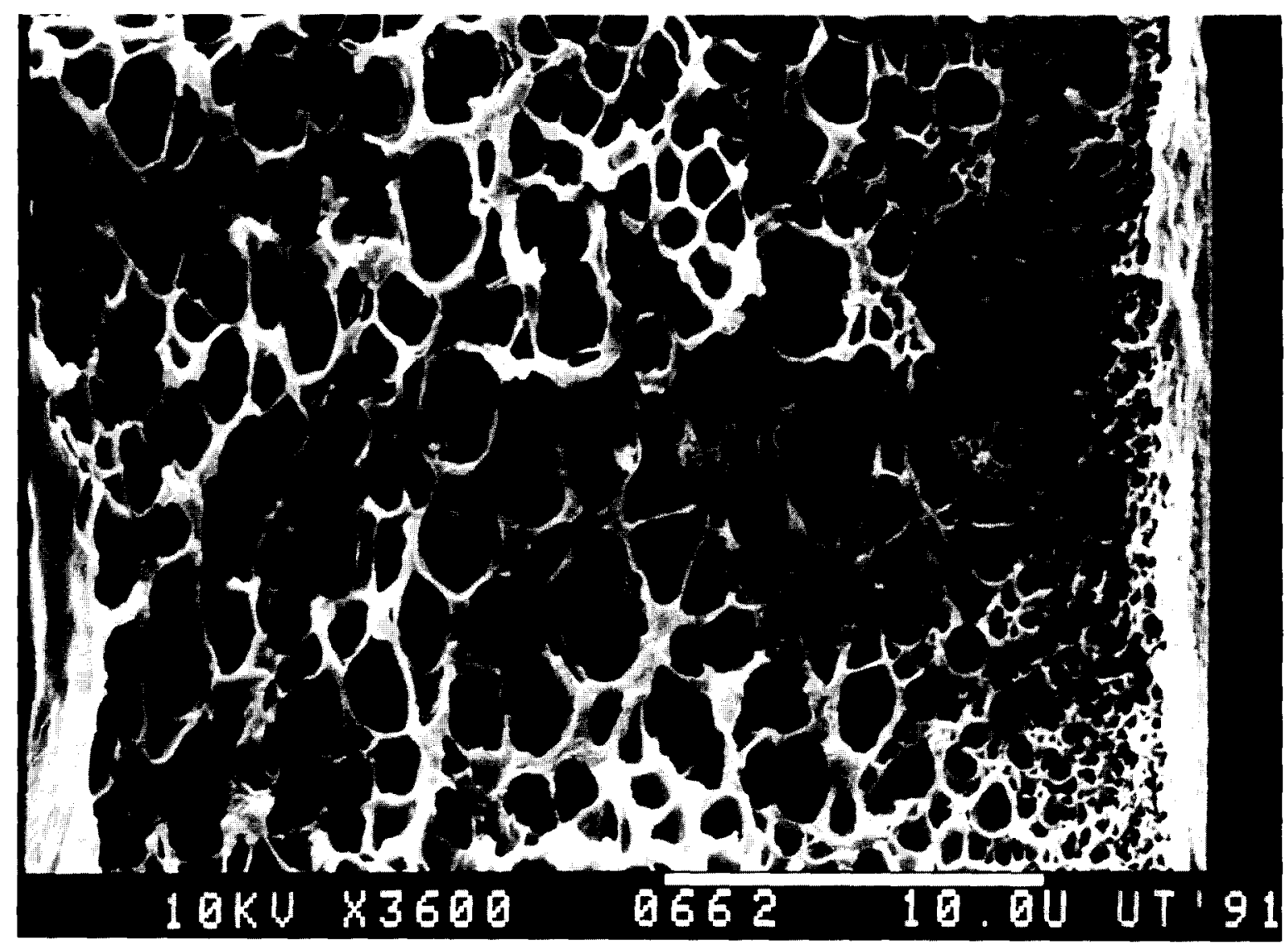

Fig. 6. Scanning electron micrograph showing details of the structure close to the outer surface of the fibre prepared from the system PLLA-dioxane-water ( system A).

were dried overnight in vacuo at ambient temperature. Cross-sections and top surfaces were transferred to a Balzers Union sputter unit, provided with a gold layer and examined using a JEOL 35 CF Scanning Electron Microscope. Thermal analysis of the samples was performed on a Perkin Elmer DSC-7 differential scanning calorimeter equipped with a Perkin-Elmer PE7500 professional computer and TAS-7 software. Cyclohexane, gallium and indium were used as calibration standards. Samples of 5-10 mg were cut from the fibres and heated in sealed DSC pans at a scan rate of $10^{\circ} \mathrm{C} / \mathrm{min}$.

\section{Phase diagrams}

To gain more insight in the demixing processes of solutions of PLLA in dioxane/water and chloroform/methanol mixtures an attempt was made to construct the ternary phase diagrams of these combinations at $25^{\circ} \mathrm{C}$. Polymer, solvent and nonsolvent were carefully weighed into pyrex glass tubes (diameter $8 \mathrm{~mm}$, total amount $2 \mathrm{~g}$ ). The tubes were subsequently frozen in liquid nitrogen and sealed under vacuum. The tubes were homogenized while rotating at $85^{\circ} \mathrm{C}$. Cloud points were obtained by slowly cooling the homogenous polymer solutions with varying polymer concentrations and solvent-nonsolvent ratios (cooling rate $0.1^{\circ} \mathrm{C} / \mathrm{min}$ ). Turbidity was detected using a Helium-Neon laser (Spectra Physics Stabilite ${ }^{\mathrm{TM}}$, model 120). Cloud points at $25^{\circ} \mathrm{C}$ were obtained by interpolation of the cloud point curves of solutions with constant solventnonsolvent ratio and varying polymer concentrations. Swelling values of PLLA in the nonsol- 


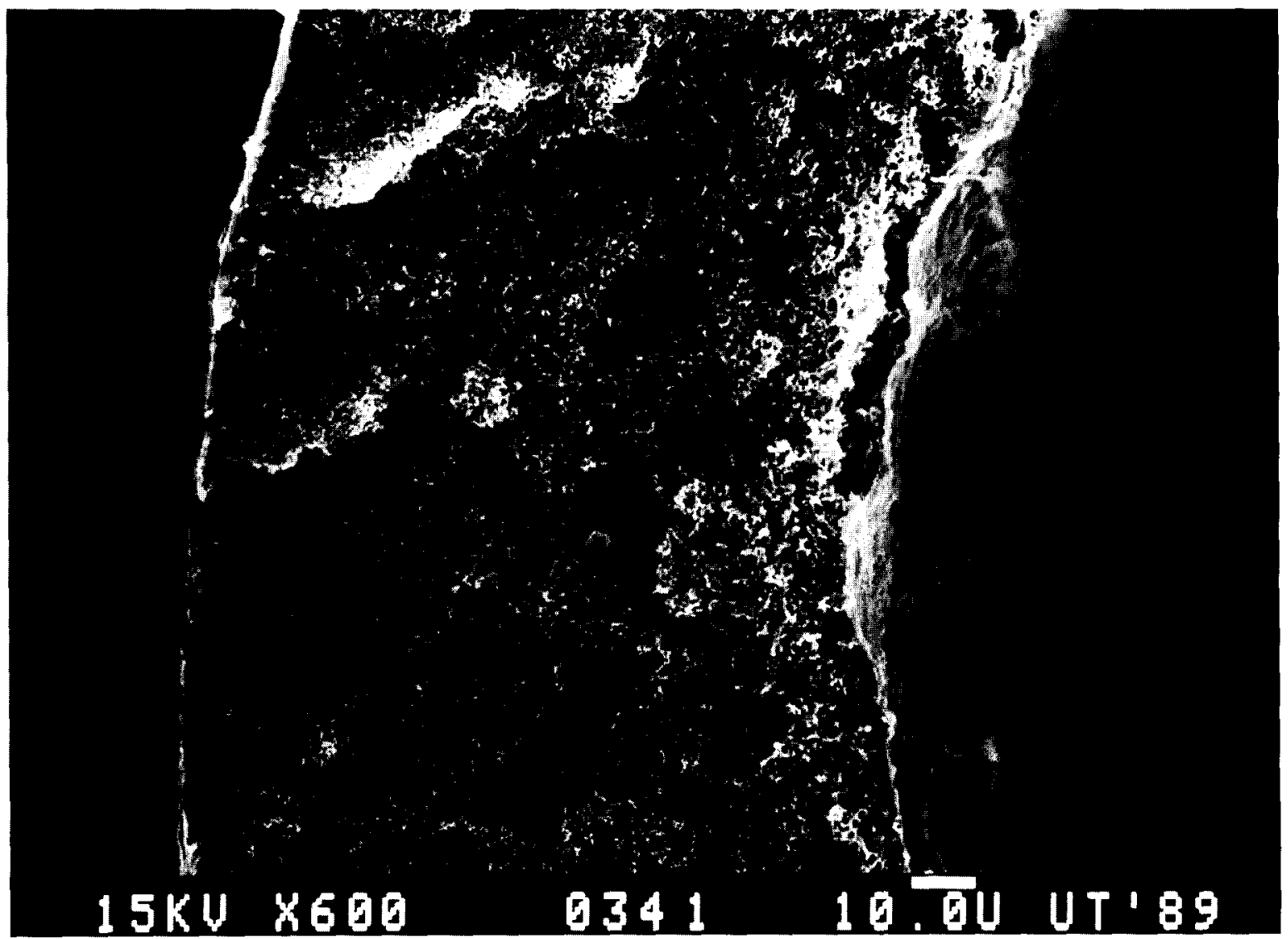

Fig. 7. Scanning electron micrograph of a cross-section of a PLLA hollow fibre membrane. Outer toplayer at the left hand side. Spinning system: PLLA-chlor $/$ tol $(65 / 35 \mathrm{v} / \mathrm{v}$ or $50 / 50 \mathrm{v} / \mathrm{v})$-meth; dissolution proc. I (systems B1 and B2).

vent were obtained by measuring the weight increase of a polymer film when exposed to the nonsolvent. The polymer films were prepared by casting a polymer solution on a glass plate and subsequently evaporating the solvent until constant weight was attained (heat of fusion of the films before immersion was $46 \mathrm{~J} / \mathrm{g}$ ). The swelling was calculated on the basis of the weight of redried films.

\section{In vitro release experiments}

\section{In vitro release of 3-ketodesogestrel}

Pre-wetted hollow fibre samples $(n=3)$ were filled with a $35 \mathrm{wt} \%$ steroid dispersion in a 20 wt $\%$ solution of PVA in water, using a syringe which was inserted into the lumen of the hollow fibre. The filled hollow fibre subsequently was heat sealed at both ends at a length of approximately $4 \mathrm{~cm}$ using a pair of hot pincers $(T=180$ $200^{\circ} \mathrm{C}$ ). Each sample of known length was immersed in a separate glass vial containing distilled water as a receiving medium (usually 50 $250 \mathrm{ml}$ ). The sealed vials were placed in a shaking water bath at $37^{\circ} \mathrm{C}$ and agitated at a frequency of $90 \mathrm{rpm}$. Once a day or once a week, depending on the release rate, the concentration of the steroid in the receiving fluid was determined using UV spectroscopy (Uvikon 930, Kontron Instruments, $\lambda=247 \mathrm{~nm}$ ). The receiving fluid of each vial was changed with such a 


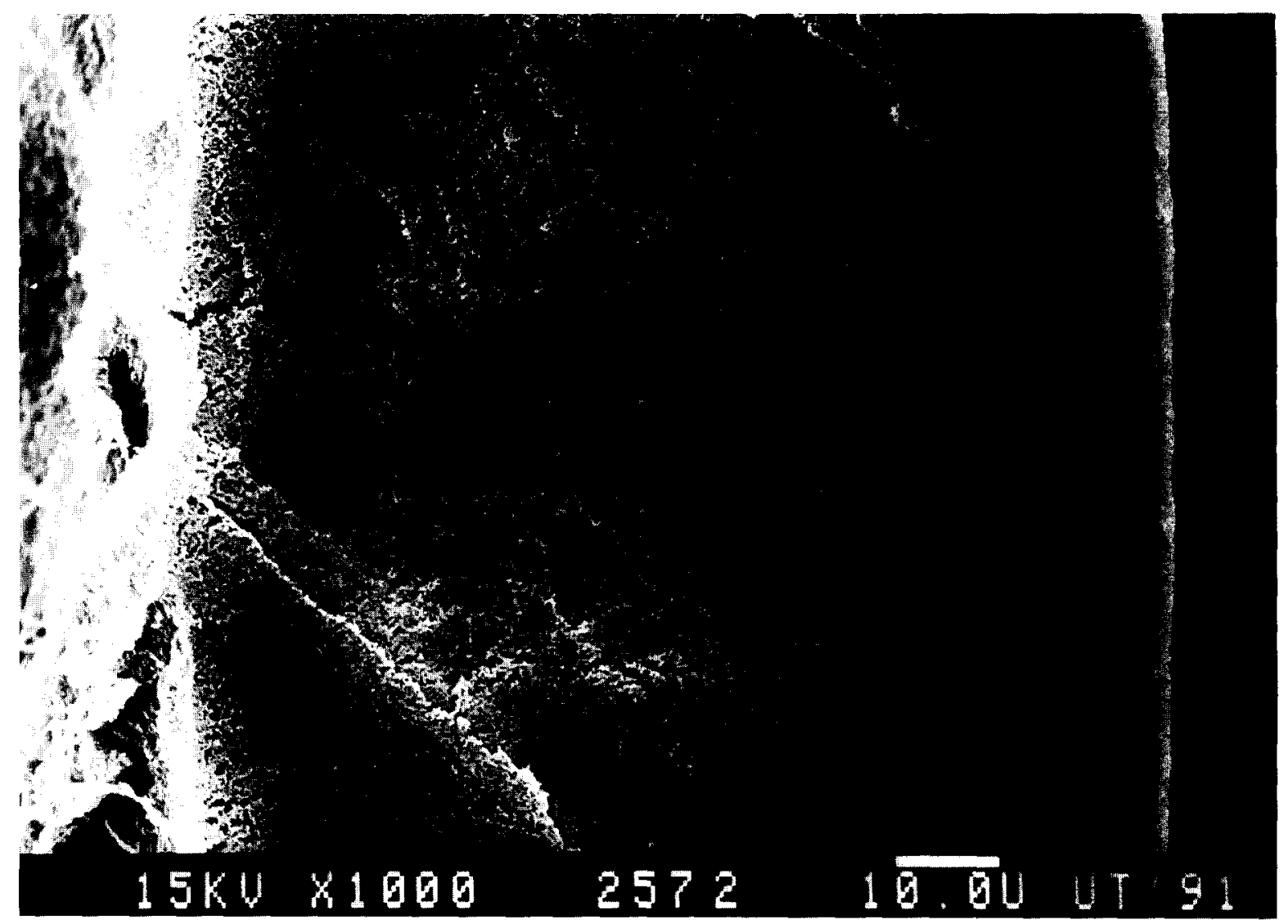

Fig. 8. Scanning electron micrograph of a cross-section of a PLLA hollow fibre membranes. Outer toplayer at the right hand side. Spinning system: PLLA-chlor/tol $(50 / 50 \mathrm{v} / \mathrm{v})$-meth; dissolution proc. II (syst. B3).

frequency that the concentration of 3-ketodesogestrel was maintained below $5 \%$ of its solubility in water.

\section{In vitro release of cisplatin}

Hollow fibre samples $(n=3)$ were filled with either a 25 or $50 \mathrm{wt} \%$ dispersion of cisplatin in a 20 to $10 \mathrm{wt} \%$ solution of PVA respectively, in a physiological saline solution $(0.9 \% \mathrm{w} / \mathrm{v})$. The fibres then were heat sealed at both ends. In vitro release of cisplatin was studied by incubating each device in a $0.1 \mathrm{M}$ phosphate buffer solution $(50 \mathrm{ml}, \mathrm{pH} 7.4)$ at $37^{\circ} \mathrm{C}$. In order to maintain sink conditions, at regular time intervals (generally each $24 \mathrm{~h}$ ) the receiving fluid was replaced. Aftcr collecting the cisplatin containing media, amounts of $1 \mathrm{M} \mathrm{NaCl}$ and $\mathrm{HCl}$ solution were added in order to obtain a $0.15 \mathrm{M} \mathrm{NaCl}$ and $0.20 \mathrm{M} \mathrm{HCl}$ concentration. Subsequently the samples were analysed for platinum content by Atomic Absorption Spectrophotometry (Varian, Techtron Spectrophotometer) [20]. The spectrophotometer was used to monitor the platinum line at $266 \mathrm{~nm}$. The sample was ionized in a flame of a mixture of air and acetylene (volume ratio 4/0.6). Release of cisplatin was measured for a period up to 7 days. 


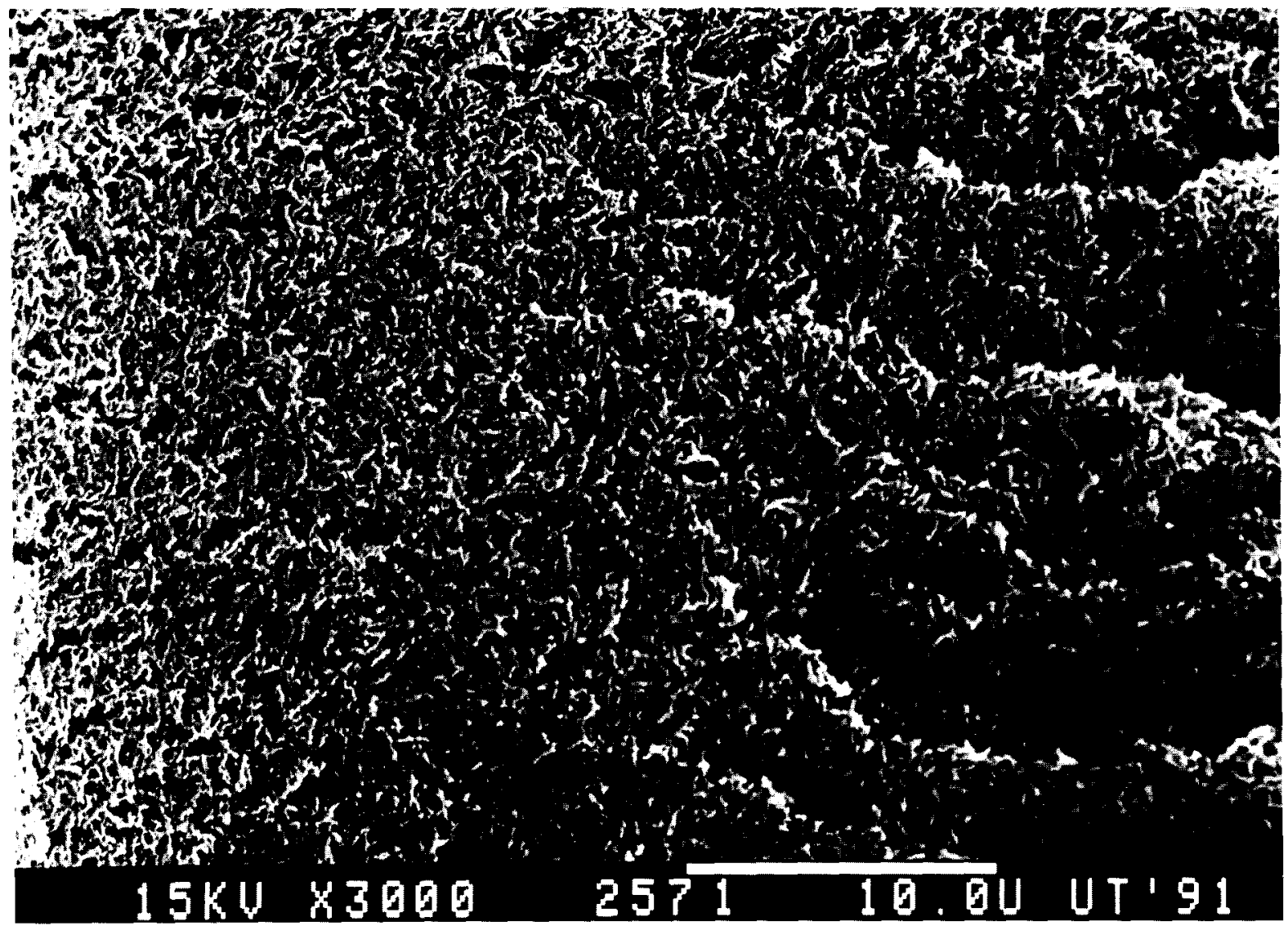

Fig. 9. Scanning electron micrograph showing details of the cross-section of a fibre prepared from the system PLLA-chlor/tol $(50 / 50 \mathrm{v} / \mathrm{v})$-meth; dissolution proc. II (system B3).

\section{In vitro release of adriamycin}

Loading of pre-wetted fibres ( $n=2$; spun from system B1 see results) with adriamycin (Adx) was carried out by immersing pieces of fibre with a length of $10 \mathrm{~cm}$ in solutions of $\mathrm{Adx}$ in saline ( 5 $\mathrm{ml} 0.9 \% \mathrm{w} / \mathrm{v} \mathrm{NaCl}$ in water, $\mathrm{pH} 5.7$ ) for $72 \mathrm{~h}$ at $37^{\circ} \mathrm{C}$. The starting concentration of Adx was varied from 5 to $200 \mu \mathrm{g} / \mathrm{ml}$. Drug loading was performed in polypropylene vessels [21], placed in a shaking bath (shaking frequency 90 cycles/ $\min$ ). To avoid photolytic inactivation of $\mathrm{Adx}$, solutions were protected from light [22].

The amount of Adx incorporated in the porous structure was calculated from the adriamycin depletion of the loading solution. The residual amount of Adx in the loading solution was determined as a function of time using reversed phase HPLC in combination with fluorescence spectroscopy $\left(\lambda_{\mathrm{ex}}=488 \mathrm{~nm} ; \lambda_{\mathrm{em}}=560 \mathrm{~nm}\right.$, Perkin Elmer LS-3 Fluorescence Spectrometer, Column: Merck 50942, LiChro Cart 125-4, LiChrospher $100 \mathrm{RP}-8,5 \mu \mathrm{m})$. Elution was performed at $25^{\circ} \mathrm{C}$ using an eluent of $30 \% \mathrm{v} / \mathrm{v}$ acetonitrile in water, $\mathrm{pH} 2.25$, flow rate: $1 \mathrm{ml} / \mathrm{min}$.

Immediately after loading, fibres were rinsed for $15 \mathrm{~s}$ in release medium to remove all adherent droplets of Adx solution. Subsequently the fibres (heat sealed at both ends) were placed in fresh saline $(50 \mathrm{ml}, 0.9 \% \mathrm{w} / \mathrm{v} \mathrm{NaCl}$ in water, $\mathrm{pH}$ 5.7 , polypropylene vessels) at $37^{\circ} \mathrm{C}$. During release, the system was shaken at a frequency of 90 cycles $/ \mathrm{min}$. After periods of $24 \mathrm{~h}$, the receiving fluid was refreshed. The highest concentration of Adx in the receiving medium did not exceed 1.4 


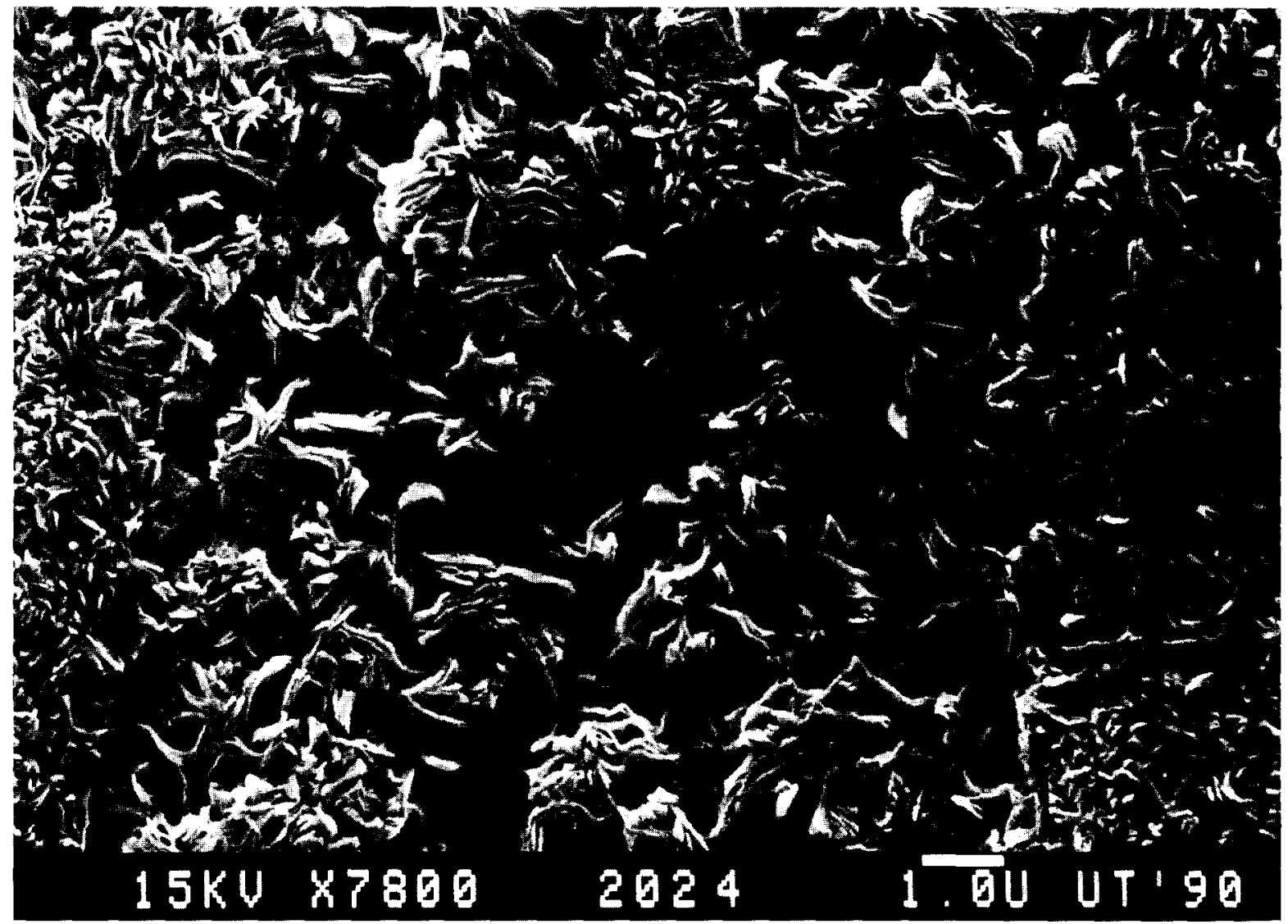

Fig. 10. Scanning electron micrograph showing details of the outer surface of a fibre prepared from the system PLLA chlor/tol $(50 / 50 \mathrm{v} / \mathrm{v})$-meth; dissolution proc. II (system B3).

$\mu \mathrm{g} / \mathrm{ml}$. Release of Adx was measured for at least 7 days. The amount of Adx released was determined as a function of time using reversed phase HPLC in combination with fluorescence spectroscopy.

\section{Results}

Microstructure and thermal properties of PLLA hollow fibre

From the spinning experiments PLLA hollow fibres are obtained with outside diameters in the range of $750-1100 \mu \mathrm{m}$ and wall thicknesses in the range of $90-130 \mu \mathrm{m}$. All combinations used showed a delay time before coagulation of the solutions occurred, except the fibres from the spinning system PLLA-dioxane-water which showed instantaneous demixing. From the system PLLA-dioxane-water (system A), asymmetric fibres were prepared consisting of a porous sublayer covered by relatively dense inner and outer toplayers (thicknesses less than $1 \mu \mathrm{m}$; Figs. 5 and 6 ). The sublayer contains small spherical pores with diameters of ca. $0.5-3 \mu \mathrm{m}$ and large voids with diameters up to $70 \mu \mathrm{m}$. Pores are poorly interconnected. From the systems B1 and B2 (PLLA-chlor/tol-meth; diss. proc. I), asymmetric fibres are obtained composed of a highly porous sublayer containing spherical pores of diameters up to $3 \mu \mathrm{m}$ (Fig. 7 ). These pores are very well interconnected. The outer surface 


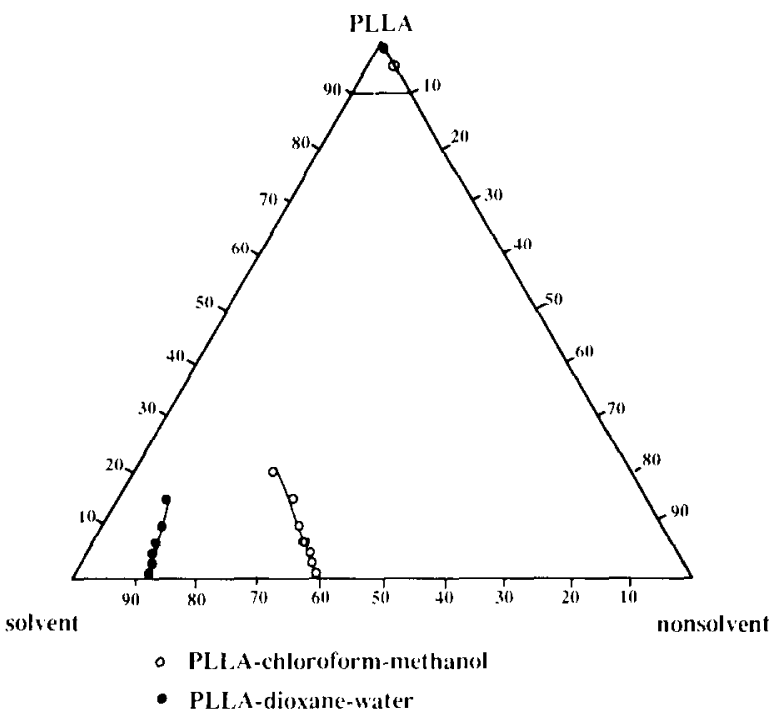

Fig. 11. Cloud points at relatively low polymer concentrations of the ternary systems PLLA-dioxane-water and PLLAchloroform-methanol. All percentages are weight percentages. Temperature: $25^{\circ} \mathrm{C}$. The glass transition is indicted with a solid line.

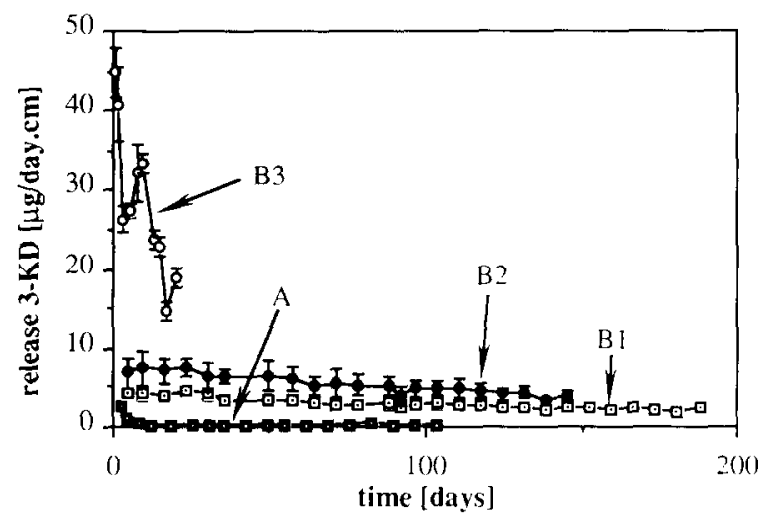

Fig. 12. Daily release of 3-ketodesogestrel in water at $37^{\circ} \mathrm{C}$ as a function of time for different types of fibres. The spinning systems are indicated in the figure. A, PLLA-dioxanewater; B1, PLLA-chloroform/toluene $65 / 35 \mathrm{v} / \mathrm{v}$-methanol diss. proc. I. B2, PLLA-chloroform/toluene $50 / 50 \mathrm{v} / \mathrm{v}$ methanol diss. proc. I. B3, PLLA-chloroform/toluene $50 / 50$ $\mathrm{v} / \mathrm{v}$-methanol diss. proc. II.

contains small pores of $0.1 \mu \mathrm{m}$. From system B3 (PLLA-chlor/tol (50/50 v/v)-meth; diss. proc. II) a completely microporous fibre can be pre-

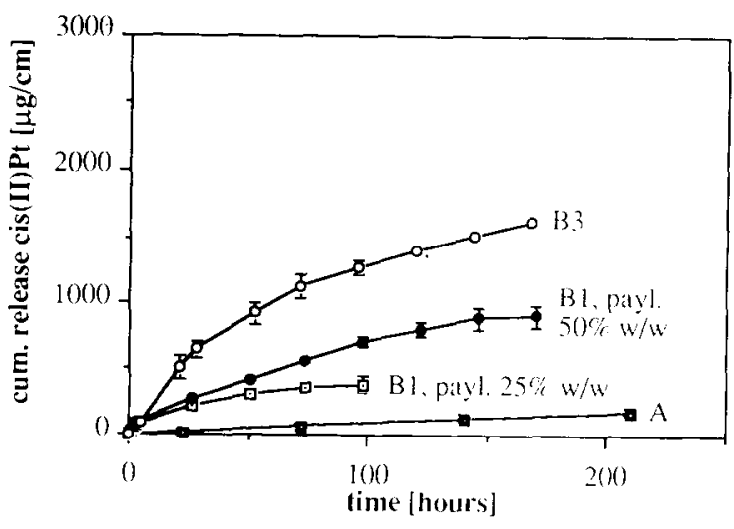

Fig. 13. Cumulative release of cisplatin in a $0.1 \mathrm{M}$ phosphate buffer solution $(\mathrm{pH}=7.4)$ at $37^{\circ} \mathrm{C}$ as a function of time for different types of fibres. Fibres spun from the system PLLAchlor/tol $(65 / 35 \mathrm{v} / \mathrm{v})$-meth (system Bl), were loaded with $25 \% \mathrm{w} / \mathrm{w}$ or $50 \% \mathrm{w} / \mathrm{w}$ cisplatin dispersions. All other fibres were loaded with $50 \% \mathrm{w} / \mathrm{w}$ cisplatin dispersion. A. PLLAdioxane-water: B1, PLLA-chloroform/toluene $65 / 35 \mathrm{v} / \mathrm{v}$ methanol diss. proc. I. B3. PLLA-chloroform/toluene 50/50 $\mathrm{v} / \mathrm{v}$-methanol diss. proc. II.

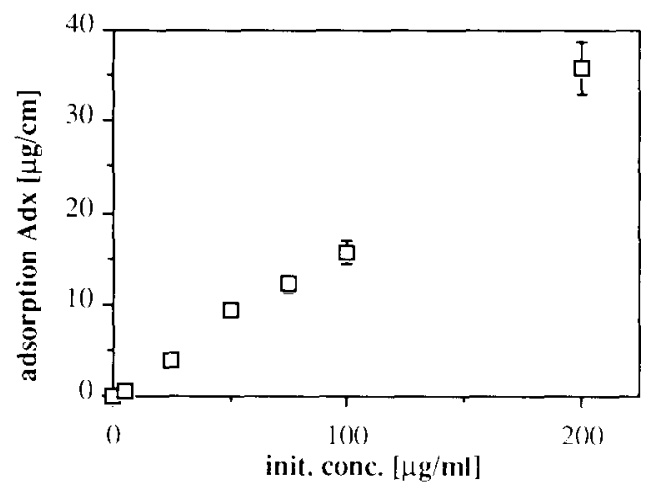

Fig. 14. Adsorption of adriamycin on porous PLLA fibres spun from the system PLLA-chlor/tol $(65 / 35 \mathrm{v} / \mathrm{v})$-meth (diss. I, system B1). Fibres with a length of $10 \mathrm{~cm}$ were immersed in solutions of adriamycin with different concentrations ( $5 \mathrm{ml}$ solution of $\mathrm{Adx}$ in saline; $\mathrm{pH}=5.7 ; T=37^{\circ} \mathrm{C}$ ).

pared (Fig. 8). The sublayer contains irregularly shaped pores with sizes smaller than $1 \mu \mathrm{m}$ (detail sublayer shown in Fig. 9). The outer and inner toplayer consist of an irregular intertwined network of 'leafy' structures (details outer surface shown in Fig. 10).

Analysis of the dry membranes by DSC shows 


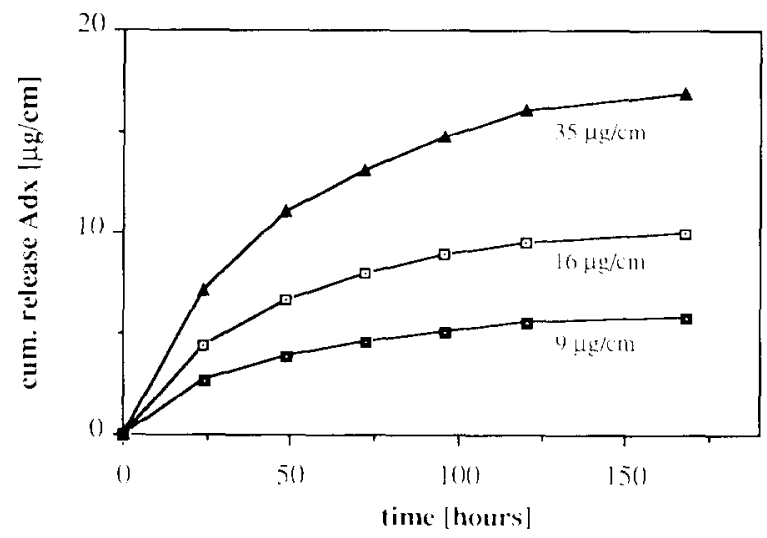

Fig. 15. Cumulative release of adriamycin from fibres spun from the systcm PLLA-chlor/tol $(65 / 35 \mathrm{v} / \mathrm{v})$-meth (diss. I, system B1). Fibres with a length of $10 \mathrm{~cm}$ and different payloads $(9,16$ and $35 \mu \mathrm{g}$ per $\mathrm{cm}$ length of the fibre, respectively) were immersed in a receiving medium of $50 \mathrm{ml}$ saline $\left(\mathrm{pH}=5.7 ; T^{\circ}=37^{\circ} \mathrm{C}\right)$.

that all membranes exhibit a melting transition upon heating. The peak temperature is in the range of $176-181^{\circ} \mathrm{C}$. All membranes are highly crystalline. Fibres from system A generally exhibit a heat of fusion of 36 to $43 \mathrm{~J} / \mathrm{g}$. For fibres from systems B1 and B2 heats of fusion of ca. 47 to $51 \mathrm{~J} / \mathrm{g}$ are found. The highest heat of fusion of $61 \mathrm{~J} / \mathrm{g}$ is found for fibres spun from system B3. The heats of fusion correspond to crystallinities between 60 and $80 \%$ using the value for $100 \%$ crystalline PLLA $(81 \mathrm{~J} / \mathrm{g}$ ) as given by Fischer et al. [28].

\section{Phase diagrams}

Because of the polydispersity of the polymer samples and possible interference of crystallization processes it is not allowed to identify the obtained curves with the binodal. The curves are therefore referred to as cloud point curves. Parts of the phase diagrams of the two ternary systems at $25^{\circ} \mathrm{C}$ are represented in Fig. 11. Some differences can immediately be noted from this figure. The curve obtained for the combination PLLAchloroform-methanol shows a slight tendency to bend to the polymer solvent axis. In addition much more methanol is needed for the demixing of solutions of PLLA in chloroform than water is needed for the demixing of solutions of PLLA in dioxane. Large differences exist for the swelling of PLLA in both nonsolvents. Maximum swelling of the polymer was already attained after 48 $\mathrm{h}$. The swollen polymer films contained $6 \% \mathrm{w} / \mathrm{w}$ methanol. The content of water was smaller than $1 \% \mathrm{w} / \mathrm{w}$.

\section{In vitro release experiments}

Figure 12 shows the daily amounts of $3-K D$ released from different PLLA hollow fibres. Fibres spun from the system PLLA-dioxane-water release the steroid at a very constant rate of 0.4 to $0.5 \mu \mathrm{g} / \mathrm{day} \cdot \mathrm{cm}$. Fibres prepared from the system PLLA-chlor/tol-methanol (chlor/tol ratio 65/35 and $50 / 50 \mathrm{v} / \mathrm{v}$, respectively; dissolution procedure I) release the drug at a much higher rate (ca. 3 and $5 \mu \mathrm{g} /$ day $\cdot \mathrm{cm}$ both after 150 days ). The release rate decreases slightly as a function of time. Fibres spun from solutions of PLLA in a chloroform/toluene mixture (ratio $50 / 50 \mathrm{v} / \mathrm{v}$ ) prepared according to dissolution procedure II, show a very fast release of steroid. The release rate fluctuates and rapidly decreases from 45 to 19 $\mu \mathrm{g} /$ day $\cdot \mathrm{cm}$ after 30 days.

Figure 13 shows the cumulative amount of cisplatin released from fibres from system B 1 , filled with 25 or $50 \mathrm{wt} \%$ dispersions of cisplatin, respectively. The fibre loaded with a $25 \mathrm{wt} \%$ dispersion of cisplatin releases the total payload of ca. $400 \mu \mathrm{g}$ per $\mathrm{cm}$ length of the fibre within 4 days. If the payload is increased by a factor of 2 , constant daily release rates are obtained up to 6 days. The influence of the fibre membrane structure on the release rate of cisplatin is also illustrated in Fig. 13. Fibres prepared from the system PLLA-dioxane-water, release the drug at a constant rate of $25 \mu \mathrm{g} /$ day $\cdot \mathrm{cm}$. The release from fibres prepared from the system PLLA-chlor/tolmeth is much higher. A constant daily release of $115 \mu \mathrm{g} /$ day $\cdot \mathrm{cm}$ is observed within 7 days for fibres from systems B1 and B2 whereas fibres from system B3 release up to $1600 \mu \mathrm{g} / \mathrm{cm}$ in the same period of time. The release rate in the latter case however decreases as a function of time.

Upon immersing porous PLLA fibre membrancs from system $\mathrm{B} 1$ in a solution of $\mathrm{Adx}$ in 
saline, Adx is taken up by slow diffusion into the membrane structure and adsorption onto the PLLA surface. Adsorption of Adx initially is very fast but due to depletion of the loading solution, the adsorption rate decreases rapidly. After $72 \mathrm{~h}$ equilibrium is reached and no further uptake of Adx could be measured. In Fig. 14 the payload (per $\mathrm{cm}$ length) is given as a function of the initial concentration of the loading solution. After transferring Adx loaded fibres to fresh saline, the adsorbed Adx gradually desorbs from the membrane surface. In Fig. 15 it is shown that the release rate increases with increasing payload of the fibre. Release rates however are not constant but continuously decrease as a function of time. After 7 days ca. $50 \%$ of the initial amount of Adx present in the membrane has been released. From that time also cleavage products of Adx (aglycon ) could be detected [22].

\section{Discussion}

As previously reported [5], the dry-wet spinning process can be used succesfully to produce PLLA hollow fibres for the controlled release of drugs. In the present study the same process was applied to prepare porous PLLA fibres suitable for release of 3-ketodesogestrel, cisplatin or adriamycin. Two different spinning systems were investigated: PLLA-dioxane-water and PLLAchloroform/toluene-methanol. Both combinations showed a delay of demixing (the spinning system PLLA-dioxane-water also shows delay of demixing when no nonsolvent is added to the spinning solution, the solution used already has a composition very close to the miscibility gap ).

The large differences in location of the cloud point curves for the systems PLLA-dioxane-water and PLLA-chloroform-methanol can in principle be explained on the basis of the Flory-Huggins theory for ternary polymer solutions $[8,25,14]$. The location of the cloud point curves depends on the three binary interaction parameters between the three components, e.g., the polymer-solvent interaction, the polymer-nonsolvent interaction and the solvent-nonsolvent interaction. Values for the three parameters are not available at the moment. It has been shown that the most dominating parameter is the polymer-nonsolvent interaction parameter [25]. The swelling values can be used as an indication for the polymer-nonsolvent interaction. From the results it appears that the interaction between PLLA and methanol is much better than the interaction between PLLA and water, in other words methanol is a worse nonsolvent than water. It is not unlikely that this difference in nonsolvent quality can explain at least partly that much more methanol is needed to demix a solution of PLLA in chloroform than water is needed to demix a solution of PLLA in dioxane. From Fig. 11 it appears that the cloud point curve for the combination PLLA-chloroform-methanol bends to the polymer-solvent axis. This could be an indication that crystallization is responsible for the turbidity at higher polymer concentrations. To verify this hypothesis, kinetic measurements should be performed. The combination PLLAchloroform-methanol has also been used as a spinning system [5]. The structures that were obtained were comparable to the system PLLAdioxane-water and showed also dense skins, the permeability of the fibres for the different types of drugs was consequently very low. Addition of toluene results in a remarkably improvement of the release characteristics. The influence of the toluene in the spinning solution on the membrane formation process is difficult to predict. PLLA cannot be dissolved in toluene itself directly and therefore toluene must be regarded as a poor solvent. Chloroform is a very good solvent for PLLA. The combination PLLA-chloroform-toluene has earlier been used for the dry spinning of fibres by Leenslag et al. [27]. The PLLA in their study however had a much higher molecular weight than the PLLA used in this study. It was noted that the preparation procedure of the spinning solution influenced the properties of the fibres. Homogeneous solutions could not be obtained by directly dissolving the polymer in the solvent mixture, but only by dissolving the polymer in chloroform and subsequently adding the toluene. The chloroform/toluene ratio was another important variable. Also, 
in this process, highly crystalline porous fibres were obtained. On the basis of measurements of the intrinsic viscosity and the optical rotation of the polymer as a function of the toluene/chloroform ratio in solution, it was suggested that at higher toluene fractions (close to the theta conditions) the polymer chain transformed from a random coil to an interrupted helix structure. The helical structure could form an ideal precursor for subsequent crystallization processes.

From experiments performed by Triolo [24] showing the glass transition of PLLA films as a function of the percentage of dichloromethane present in the film, an indication can be obtained for the solvent concentration in the film necessary to decrease the glass transition temperature from $60^{\circ} \mathrm{C}$ (glass transition of pure PLLA) to $25^{\circ} \mathrm{C}$. It was found that $8-10 \% \mathrm{w} / \mathrm{w}$ solvent was required to reach a glass transition of $25^{\circ} \mathrm{C}$. The glass transition is probably located at polymer concentrations that are too high to play an important role in the initial stages of the membrane formation process.

Regarding the shape of the pores of fibres from the system PLLA-dioxane-water it is apparent that the membrane structure is formed by nucleation and growth of polymer poor droplets. The membranes were highly crystalline and crystallization processes probably play an important role during the fixation of the structure. As discussed in the theory section toplayers with a relatively dense structure may have been formed because of a strong increase of the polymer concentration in the polymer solution close to the coagulation bath. The top layers were thinner than the top layers of the fibres spun with spinning solutions that did not contain water [5].

The same mechanism of phase separation may have occurred for membrane formation from the systems B1 and B2. Fibres from the system B3 (PLLA-chlor/tol $(50 / 50 \mathrm{v} / \mathrm{v})$-meth; diss. proc. II) have a structure very different compared to the other systems. At this moment no explanation is available by which mechanism the presence of toluene in the solution and the applied dissolution procedure influence the fibre structure. It seems very likely that the reported influ- ence of the toluene on the crystallization of PLLA plays an important role during the structure formation. However also other unknown parameters like the exchange rates of both solvents for methanol and the homogeneity of the polymer solution will almost certainly have an influence on the fibre structure.

It has been shown that release characteristics of the different compounds can be adjusted adequately by variation of the membrane structure. For the release of the steroid or the cytostatic agents, diffusion of the compound through a well interconnected porous structure is needed as the permeability of the dense polymer for the drugs is very low. Release of steroid and cisplatin could be succesfully established by loading the device with solid particles thus providing a unit activity in the fibre core. Generally a constant release rate could be obtained which implies that the membrane is rate-controlling. The very high release rates of the fibres from the system $B 3$ cannot be explained by the fact that the outer diameters of this type of fibre are somewhat larger compared to those of fibres prepared following other procedures (outer diameters 750 and $1100 \mu \mathrm{m}$, respectively). The observed decrease of release rates with these very porous fibres suggests that in this case the membrane is no longer rate controlling. A reason for the observed decrease and fluctuations of the release rate may be a considerable reduction of the concentration gradient across the fibre core formulation caused by a low rate of dissolution of steroid crystals in the core vehicle. Because drug particle sizes are not completely uniform, a declining dissolution rate may arise due to depletion of smaller drug particles in the initial stages of the release process. Also, aggregation of drug particles and drug recrystallization, producing an increase in particle size, may cause a decrease and fluctuations in the rate of dissolution [23].

Release of adriamycin could be obtained through an adsorption-desorption mechanism. The fibre could be loaded by diffusion of the drug into the porous network and adsorption onto the internal pore surface presumably by hydrophobic interaction [21]. As the adsorption process 
appeared to be reversible, the drug could be released from the fibre. The mechanism of release of adriamycin from the porous fibre wall is complex. By desorption, the drug is released from the pure surface. After desorption the drug can be released from the device by diffusion through the porous network. As may be clear the release rate of the drug is governed by the desorption rate of the drug from the free surface, the concentration of free drug in the pores and the rate of diffusion through the pores. The latter process is influenced by the structure of the fibre wall. At this moment there are indications that the rate of desorption of the drug from the surface is not the rate-limiting step in the release process. A thorough study of adsorption, desorption and diffusion processes is needed to provide a full description of the release mechanism. By a proper choice of payload and membrane structure, a release device with optimal release properties may be obtained.

\section{Conclusion}

By adjustment of system and process parameters it is possible to obtain highly crystalline asymmetric PLLA fibres. Fibre membranes prepared from the system PLLA-dioxane-water consist of a porous layer covered by relatively dense inner and outer toplayers. Liquid-liquid demixing probably dominates the structure formation process. Crystallization takes place after a sufficicntly concentrated polymer rich phase is formed. From the system PLLA-(chloroform/ toluene)-methanol it is possible to prepare completely microporous membranes. There are indications that in the latter case crystallization of PLLA during membrane formation strongly influences the ultimate structure of the fibre wall.

Hollow fibres are suitable for long term zeroorder in vitro release of the contraceptive 3-ketodesogestrel and short term zero-order release of cisplatin. The drugs are released by dissolution of the solid drug crystals in the fibre core followed by diffusion through the membrane pores. Release characteristics are influenced by the membrane structure. Short term release of adri- amycin from porous PLLA fibres could be obtained through an adsorption-desorption mechanism. Fibres loaded with relatively low amounts of adriamycin show a sustained in vitro release. Both the rates of desorption of the drug and diffusion processes may determine the overall release characteristics of the system.

\section{Acknowledgements}

The authors like to thank Dr R. Verrijk and Dr A.C. Begg of the Netherlands Cancer Institute for their contributions with the release experiments.

\section{References}

1 J. Heller, Biodegradable polyners in controlled drug delivery, CRC Crit. Rev. Ther. Drug Delivery Systems 1 (1984) 39-90.

2 S.J. Holland, B.J. Tighe and Ph.L. Gould Polymers for biodegradable medical devices. 1. The potential of polyesters as controlled macromolecular release systems, J. Contr. Rel. 4 ( 1986 ) 155-180.

3 R. Duncan and L.W. Seymour, Controlled release technologies: a survey of research and commercial applications, Elsevier Science Publishers, Amsterdam (1989).

4 European Patent Application no. 86402527.5, Porous bioabsorbable polyesters (A. Schindler, Research Triangle Institute, Durham, North Carolina, US ) 1987

5 M.J.D. Eenink, J. Feijen, J. Olijslager, J.H.M. Albers, J.C. Rieke and P.J. Greidanus, Biodegradable hollow fibres for the controlled release of hormones, J. Controlled Release 6 ( 1987 ) 225-247.

6 J. Aubert, A. Sylwester and P. Rand, Microcellular polymer foams for controlled release and as biomaterials. Polymer Preprints, 30 (1989) 447-448.

7 A.D. Schwope and D.L. Wise, Evaluation of wound covering materials, J. Biomed. Mater. Res. 11 (1977) 489-502.

8 P. Flory, Principles of polymer chemistry, Cornell University Press, Ithaca, New York, 1953.

9 O. Olabisi, L.M. Robeson and M.T. Shaw, Polymerpolymer miscibility, Academic Press, New York, 1981.

$10 \mathrm{~J}$. Arnauts and H. Berghmans, Amorphous thermoreversible gels of atactic polystyrene, Polym. Commun., 28 (1987) 66-68

11 R.M. Hikmet, S. Callister and A. Keller, Thermoreversible gelation of atactic polystyrene: phase transformation and morphology, Polymer 29 (1988) 1378-1388.

12 D.R. Lloyd, K.E. Kinzer and H.S. Tseng, Microporous membrane formation via thermally induced phase separation. I. Solid-liquid phase separation, J. Membr. Sci. 52 ( 1990$) 239-261$. 
13 W.R. Burghardt, Phase diagrams for binary polymer systems exhibiting both crystallization and limited liquid-liquid miscibility, Macromolecules 22 (1989) 24822486.

14 A.J. Reuvers, J.W.A. van den Berg and C.A. Smolders, Formation of membranes by means of immersion precipitation. Part I. A model to describe the mass transfer during immersion precipitation, J. Membr. Sci. 34 ( 1987 ) 45-65.

15 P. Radovanovic, S.W. Thiel and S.-T. Hwang, Formation of asymmetric polysulfone membranes by immersion precipitation. Part 1. Modelling mass transport during gelation, J. Membr. Sci. 65 (1992) 213-229.

16 C.S. Tsay and A.J. McHugh, Mass transfer modelling of asymmetric membrane formation by phase inversion, $\mathbf{J}$. Pol. Sci: Part B; PP, 28 (1990) 1327-1365.

17 G.E. Gaides and A.J. McHugh, Gelation in an amorphous polymer: a discussion of its relation to membrane formation, Polymer 30 (1989) 2118-2123.

18 W.R. Burghardt. L. Yilmaz and A.J. McHugh, Glass transition crystallization and thermoreversible gelation in ternary PPO solutions, relationship to asymmetric membrane formation, Polymer 28 ( 1987 ) 2085-2092.

19 I. Cabasso, E. Klein and J.K. Smith, Polysulfone hollow fibres. I. Spinning and properties, J. Applied Pol. Sci. 20 (1976) 2377-2394.

20 G. Spenlehauer, M. Vert, J.-P. Benoît, F. Chabot and M. Veillard, Biodegradable cisplatin microspheres prepared by the solvent evaporation method: morphology and release characteristics, J. Controlled Release 7 (1988) 217-299.
21 E. Tomlinson and L. Malspeis, Concomitant adsorption and stability of some anthracycline antibiotics. J. Pharm. Sci. 71 (1982) 1121-1125.

22 A.G. Bosanquet, Stability of solutions of antineoplastic agents during preparation and storage for in vitro assays. II. Assay methods, adriamycin and the other antitumour antibiotics, Cancer Chemother. Pharmacol. 17 (1986) $1-10$.

23 C.G. Pitt, M.M. Gratzl, A.R. Jeffcoat, R. Zweidinger and A. Schindler, Sustained drug delivery systems. II. Factors affecting release rates from poly ( $\epsilon$-caprolacone $)$ and related biodegradable polyesters, J. Pharm. Sci. 68 (1979) 1534-1538.

24 P.M. Triolo, The release of bovine serum albumin from biodegradable poly-L-lactic acid matrices, Thesis, University of Utah, 1987.

25 F. Altena, Phase separation in cellulose acetate solutions in relation to asymmetric membrane formation, Thesis, University of Twente, 1982.

26 J.G. Wijmans, J.P.B. Baaij and C.A. Smolders, The mechanism of formation of microporous or skinned membranes produced by immersion precipitation, $\boldsymbol{J}$. Membr. Sci. 14 (1983) 263-274.

27 I.W. I.eenslag and A.J. Pennings, High-strength poly-Llactide fibres by a dry-spinning hot-drawing process, Polymer 28 (1987) 1695-1702.

28 E.W. Fischer, H.J. Stcrzel and G. Wegner, Investigations of the structure of solution grown crystals of lactide copolymers by means of chemical reactions, Kolloid-Z. Z Polymere 251 (1973) 980-990. 